\title{
Performance Studies of Gap-Graded Mixes using Elastomer Modifier for Wearing Course
}

\author{
Anusha T M ${ }^{\mathrm{a}}$, Pavan Kumar $\mathrm{M}^{\mathrm{b}}$, H S Jagadeesh \\ a,b,c Department of Civil Engineering, \\ B M S College of Engineering, Bengaluru, Karnataka, India
}

\begin{abstract}
Road construction crossing the world created huge demand for construction materials. Natural aggregates is the prime material for the road construction use of aggregates leads to question about the preservation of natural resource. In other hand use of recycled pavement asphalt (RAP) as a alternative material in place natural aggregate which makes environmental friendly construction material. This research investigated the feasibility of using reclaimed asphalt pavement in stone mastic asphalt mix by modifying the binder with waste engine oil and elastomer modifier. RAP is replaced with aggregates at $10 \%, 20 \%$, $30 \% \& 40 \%$. Further in order to increase the percentage of RAP in SMA mix waste engine oil and elastomer modifier is added i.e., RAP of $50 \%, 60 \%$ and $70 \%$. The results indicated that $30 \%$ of RAP is found to be feasible through laboratory investigation for SMA mixes and $60 \%$ RAP is found to be feasible to use in SMA mix through laboratory investigation by modifying binder with waste engine oil and elastomer modifier.
\end{abstract}

Keywords: Stone matrix asphalt; Wearing course; Reclaimed asphalt pavement; Elastomer modifier; Fatigue

\subsection{Stone matrix asphalt}

\section{INTRODUCTION}

India which has an ideal vision for growth and development, of of infrastructure. Infrastructure development is a core aspect of connectivity which connects different places through different modes of transportation, wherein road connectivity is undoubtedly one of the most important mode. For a robust road connectivity network, the study of pavement structure and their durability and resistance will be crucial aspect. This makes us look at some of the key aspects like type of pavements and materials used for construction and the cost incurred to lay the pavement.

The pavements undergo repetitive loads and the number of deflections and numerous loads are imposed once a wheel moves on the surface where the load is dissipated to the underlying layers of the pavement are subjected to diverse type of distress. The major distresses found in flexible pavements across the globe are rutting and fatigue. Stone matrix asphalt is found be and effective alternative on heavy traffic pavements due to its stone on stone contact it provides resistance to rutting and higher binder content increases fatigue life and reduces reflection cracking [1].

\subsection{Reclaimed Asphalt Pavement}

Reclaimed asphalt pavement are the materials derived from reprocessed pavement which contains asphalt and aggregates. The derived materials are subjected to crushing and screening to obtain RAP of well graded aggregates coated with bitumen. Recycling of existing asphalt pavements allows the industry to produce new pavements with effective utilization of material and energy making it cost effective [7]. For this study, RAP is procured from Chittoor district where some part of BengaluruTirupati Highway (NH-206) was scarified for construction of cement concrete pavement.

\subsection{Stabilizing Additive}

SMA is a gap graded mix, contains high percentage of voids due to which the bitumen content is more. Hence, the stabilizing additive should be added to the mix to reduce the draindown characteristic of the mix [3]. In this study cellulose fiber is used as stabilizing additive.

\subsection{Mineral Filler}

Mineral filler is the material which passes through $0.075 \mathrm{~mm}$ sieve size. The major components of the filler consist of rock dust, portland cement, hydrated lime, ground limestone dust or fly ash. This makes the SMA mix stiffer and a rich binder. Breaching of the threshold level of the filler can lead to excessive stiffening of the mix causing unfavourable conditions for compaction. This might lead to the cracking of the susceptible mix. In general, aggregates passing through 0.075 $\mathrm{mm}$ sieve size should be $8-12 \%$ of the total amount of aggregate in SMA mix. The main function of filler is to fill the voids in aggregate skeleton and make the mix denser by improving the cohesion of the asphalt binder and the stability [2]. In this study, a waste material called baghouse dust is used as filler material in SMA mix.

\subsection{Elastomer Modifier}

The elastomer modifier consists of functional additive which is a smart solution to improve the properties of mix around the globe. It gives better durability, convincing anti-rutting characteristics, better aging resistance and enhanced performance. Apart from this, it is an alternative way to achieve a process of reliable modification of bituminous mix close to the same performance level with modified bitumen. In this study commercially available elastomer modifier pellets are used. This product is a pelletized blend of cellulose fiber and functional additive. The composition consists of $20 \%$ and $80 \%$ by weight respectively. The quantity of elastomer modifier is added at a rate of $12 \%$ or $15 \%$. In this study dosage rate of $15 \%$ is considered for viscosity grade 30 which is recommended in the manual [20]. The mixing of elastomer modifier with the mix is carried out by two modification procedures i.e., dry process and wet process. In this research the modification of the bitumen is carried out by adopting dry process technique.

\subsection{Objectives of the work}

- To assess the performance of stone matrix asphalt mix with partial replacement of reclaimed asphalt pavement

- To determine the feasibility of using reclaimed asphalt 
pavement in stone matrix asphalt mix.

- To determine the effect of elastomer modifier in stone matrix asphalt mix with reclaimed asphalt pavement.

\subsection{Scope of the work}

Recycling asphalt pavement initiates a cycle of $4 \mathrm{R}$ principles, where the materials can be recycled and reuse which leads to a sustainable use of natural resources. Reclaimed asphalt pavement is one such alternative to unexploited materials which can reduce the over use of limited aggregates. By using reclaimed asphalt pavement sustainability can be achieved without compromising its structural performance. As RAP is an aged material, to regain the fresh properties different commercial rejuvenators are used. There are instances wherein commercial rejuvenators are not available. Therefore, this study analyses the potential of modifying the asphalt with elastomer modifier as a substitute for commercial rejuvenators to increase the percentage of the reclaimed asphalt pavement materials which can meet the intensified needs more legitimately.

\section{LABORATORY INVESTIGATIONS}

\subsection{Aggregates}

The aggregates were procured from different crushers. The basic tests were carried out on the aggregates and it was found that aggregates procured from KMS crusher-Bagalur, Karanataka were found to be within the required limits as per MoRTH [16] for SMA mix. The basic test results are depicted in Table 1.

Table 1 Tests on aggregates

\begin{tabular}{|c|c|c|}
\hline Tests conducted & Test Results & $\begin{array}{l}\text { Specifications as per } \\
\text { MORTH 5th Revision }\end{array}$ \\
\hline Aggregate Impact Test & $16.1 \%$ & $\operatorname{Max} 18 \%$ \\
\hline $\begin{array}{c}\text { Aggregate Crushing } \\
\text { Value }\end{array}$ & $21.32 \%$ & - \\
\hline $\begin{array}{c}\text { Los Angeles Abrasion } \\
\text { Value }\end{array}$ & $17.5 \%$ & $\operatorname{Max} 25 \%$ \\
\hline $\begin{array}{l}\text { Combined Flakiness and } \\
\text { Elongation Index }\end{array}$ & $19.69 \%$ & $\operatorname{Max} 30 \%$ \\
\hline $\begin{array}{l}\text { Water Absorption } \\
\text { Specific Gravity }\end{array}$ & $0.20 \%$ & $\operatorname{Max} 2 \%$ \\
\hline $20 \mathrm{~mm}$ down & 2.68 & \\
\hline $12 \mathrm{~mm}$ down & 2.69 & Not Specified \\
\hline $\begin{array}{l}6 \mathrm{~mm} \text { down } \\
\text { Stone Dust }\end{array}$ & $\begin{array}{l}2.71 \\
2.72\end{array}$ & 1 vor spectined \\
\hline Polished Stone Value & 58 & $\operatorname{Min} 55$ \\
\hline
\end{tabular}

\subsection{Reclaimed asphalt pavement materials}

The existing Bengaluru-Tirupati Highway ( $\mathrm{NH}$ 206) from Karnataka-Andra Pradesh border to Chittoor is completely scarified including subgrade and upgraded to cement concrete pavement. The scarified pavement sections were dumped at dumping sites. The pavement was 15-20 years old as per the records. The single lane road was upgraded to 2 lanes undivided highway in the financial year 2000-2001. Hence, the old pavement was scarified. The physical tests on RAP aggregates are conducted and RAP binder is extracted by centrifuge extractor and results are tabulated as shown in Table 2.
Table 2 Tests on RAP materials

\begin{tabular}{ccc}
\hline Tests conducted & Test Results & $\begin{array}{c}\text { Specifications as } \\
\text { per MORTH 5th } \\
\text { Revision }\end{array}$ \\
\hline Aggregate Impact Test & $16.3 \%$ & Max 18\% \\
Los Angeles Abrasion Value & $19.88 \%$ & Max 25\% \\
Combined Flakiness and & $16.80 \%$ & Max 30\% \\
Elongation Index & $0.60 \%$ & Max 2\% \\
Water Absorption Test & & \\
Specific Gravity Test & 2.51 & Not Specified \\
20 mm down & 2.59 & Min 55 \\
12 mm down & 2.54 & \\
6 mm down & 60 & \\
Polished Stone Value & $3 \%$ & \\
RAP binder & & \\
&
\end{tabular}

\subsection{Bitumen}

In the present study Viscosity Grade (VG) - 30 is used, which is obtained from HINCOL Pvt Ltd, Mangalore. The tests on the bitumen is carried out as per IRC SP: 73:2013 - Guidelines for Paving Bitumen Specifications [19]. The results are tabulated in the following Table 3.

Table 3 Tests on bitumen

\begin{tabular}{ccc}
\hline Tests conducted & Test Results & $\begin{array}{c}\text { Specifications as } \\
\text { per IS 73:2013 }\end{array}$ \\
\hline $\begin{array}{c}\text { Penetration Test at } 25^{\circ} \mathrm{C}, \\
0.1 \mathrm{~mm}\end{array}$ & 64 & Min 45 \\
$\begin{array}{c}\text { Softening Point }\left({ }^{\circ} \mathrm{C}\right) \text {, min } \\
\text { Ductility Test }(\mathrm{cm}) \text { at }\end{array}$ & 48 & Min 47 \\
$25^{\circ} \mathrm{C}$ & $100+$ & Min 40 \\
Flash Point $\left({ }^{\circ} \mathrm{C}\right)$ & 320 & Min 220 \\
Fire Point $\left({ }^{\circ} \mathrm{C}\right)$ & 350 & - \\
Specific Gravity & 1.02 & $0.97-1.02$ \\
\hline
\end{tabular}

\subsection{Elastomer modifier}

In this research, a commercially available elastomer modifier is used which is the combination of cellulose fibre and functional additive. It is a patent product obtained from SMART technologies, Peenya, Bengaluru. The characteristics of the modifier are shown in Table 4.

Table 4 Characteristics of Elastomer modifier

\begin{tabular}{cc}
\hline \multicolumn{2}{c}{ Characteristics } \\
\hline Form of modifier & Pellets \\
Colour and shape & Grey and cylindrical \\
Content of cellulose fibre & $20 \%$ \\
Content of functional additive & $80 \%$ \\
Average pellet length & $3-20 \mathrm{~mm}$ \\
Average pellet thickness & $3-6 \mathrm{~mm}$ \\
Bulk density & $280-380 \mathrm{~g} / 1$ \\
Sieve analysis $(<3.55 \mathrm{~mm})$ & $\mathrm{Max} 8 \%$ \\
\hline
\end{tabular}

\subsection{Gradation of aggregates}

After the basic tests, the aggregates are further used in the determination of the proper blend to give a good mix consisting of different aggregate sizes. The different sizes of aggregates used to obtain the proper blend are $20 \mathrm{~mm}$ downsize, $12 \mathrm{~mm}$ downsize, and $6 \mathrm{~mm}$ downsize and stone dust. 4000gm of aggregates were taken for sieve analysis. The aggregate gradation is carried out using the trial and error method, to determine the individual percentages of the different size aggregates to be used in the mix and should be within the limits as per MoRTH Table 500-37 $7^{[16]}$. The sieve analysis results and 
obtained gradation of wearing course for conventional SMA mix is as shown in Table 5.

Table 5 Gradation of SMA mix $(13 \mathrm{~mm})$

\begin{tabular}{|c|c|c|c|}
\hline \multirow{2}{*}{$\begin{array}{l}\text { IS Sieve Size } \\
(\mathrm{mm})\end{array}$} & \multicolumn{2}{|c|}{$\begin{array}{l}\text { Desired Gradation as per MoRTH } \\
\text { Specifications }\end{array}$} & \multirow{2}{*}{$\begin{array}{l}\text { Obtained } \\
\text { gradation }\end{array}$} \\
\hline & Upper limit & Lower limit & \\
\hline 19 & 100 & 100 & 100 \\
\hline 13.2 & 100 & 90 & 96.875 \\
\hline 9.5 & 75 & 50 & 69.85 \\
\hline 4.75 & 28 & 20 & 22.868 \\
\hline 2.36 & 24 & 16 & 17.772 \\
\hline 1.18 & 24 & 13 & 16.404 \\
\hline 0.6 & 18 & 12 & 15.744 \\
\hline 0.3 & 20 & 10 & 14.182 \\
\hline 0.075 & 12 & 8 & 11.552 \\
\hline
\end{tabular}

\section{RESULTS AND DISCUSSIONS}

\subsection{Marshall properties}

The SMA mix design basically relies on volumetric properties such as air voids, voids in mineral aggregate, voids in the coarse aggregate and binder content. Another important consideration in designing SMA mix is the filler/additive. In this study cellulose fibre was used as stabilizing additive and baghouse dust was used as filler material. The marshall specimen are casted and compacted with 50 blows on either side as per marshall procedure. The SMA mix design shall meet0the requirements0given in Table 6 . The optimum binder content and optimum RAP content with and without elastomer modifier is obtained by casting marshall specimens for different bitumen contents. Three specimens are casted for each bitumen content. The marshall test is carried out for three SMA mixes i.e., conventional SMA mix, SMA mix with RAP without elastomer modifier and SMA mix with RAP and elastomer modifier. For Conventional SMA mix the marshall specimens were casted for $5.75 \%, 6 \%, 6.25 \%$ and $6.5 \%$ bitumen content and were tested. The optimum binder content was found to be $6 \%$. The optimum RAP was determined by replacing RAP in different percentages i.e., $10 \%, 20 \%, 30 \%, 40 \%$ and $50 \%$. The bitumen was also varied in same range as in conventional SMA mix. The marshall stability at $30 \%$ RAP replacement with $6 \%$ bitumen content was found to be highest when compared to other proportions. Hence, the optimum RAP was considered as $30 \%$. An attempt is made to enhance the optimum RAP by adding elastomer modifier at a quantity of $15 \%$ of the weight of bitumen. This elastomer modified is added to the mix by dry process method. The bitumen content of $6 \%$ obtained for $30 \%$ RAP replacement was considered for further RAP replacement of $30 \%, 40 \%$, $50 \%, 60 \%, 70 \%$ and $80 \%$. The marshall properties at $60 \%$ RAP showed better results compared to other proportions. The marshall results of all the mixes are tabulated in the Table 7.

Table 6 SMA mix requirements

\begin{tabular}{cc}
\hline Mix design parameters & Requirements \\
\hline Air Voids $(\%)$ & 4 \\
Bitumen Content $(\%)$ & Min 5.8 \\
Cellulous Fibre $(\%)$ & Min $0.3 \%$ by weight of total mix \\
VMA $(\%)$ & 17 min \\
VCA $(\%)$ & Less than VCA (dry rodded) \\
Draindown $(\%)$ & 0.3 max \\
TSR $(\%)$ & Min 85 \\
\hline
\end{tabular}

Table 7 Marshall properties of SMA mixes

\begin{tabular}{cccc}
\hline Marshall & $\begin{array}{c}\text { Conventional } \\
\text { PMA mix }\end{array}$ & $\begin{array}{c}\text { SMA mix } \\
\text { with 30\% } \\
\text { RAP }\end{array}$ & $\begin{array}{c}\text { SMA mix with } \\
60 \% \text { RAP and } \\
\text { elastomer } \\
\text { modifier }\end{array}$ \\
\hline OBC $(\%)$ & 6 & 6 & 6 \\
Gm $\left(\mathrm{g} / \mathrm{cm}^{3}\right)$ & 2.36 & 2.353 & 2.389 \\
Gt $\left(\mathrm{g} / \mathrm{cm}^{3}\right)$ & 2.46 & 2.45 & 2.49 \\
Vv $(\%)$ & 4.03 & 4.02 & 4.15 \\
Vb $(\%)$ & 13.09 & 13.06 & 13.26 \\
VMA $(\%)$ & 17.12 & 17.07 & 17.41 \\
VFB $(\%)$ & 76.48 & 76.47 & 76.17 \\
Stability $(\mathrm{kN})$ & 8.4 & 13.02 & 9.390 \\
Flow $(\mathrm{mm})$ & 3.080 & 4.04 & 4.023 \\
\hline
\end{tabular}

\subsection{Draindown test}

The draindown characteristics for the SMA mix were conducted for the conventional SMA mix as well as for the SMA mix with optimized RAP with and without elastomer modifier, the results are tabulated in Table 8.

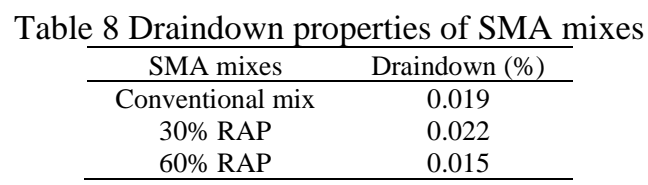

The draindown test results yielded good results, the draindown of bitumen of the specimens are found out to be well within the limit of $0.3 \%$ maximum as prescribed in MoRTH [16].

\subsection{Moisture Susceptibility}

The main factor leading to premature failure of the bituminous pavements is due to the presence of moisture in the pavement surface and the inability of aggregates to retain the coating in the presence of moisture. Hence, it is important to conduct tests regarding moisture susceptibility. Tensile strength ratio is carried out on the basis of the indirect tensile strength procedure this test is done for the conventional SMA mix as well as for the SMA mix with optimized RAP with and without elastomer modifier. The number of blows for ITS is found out at 32 blows on both sides for conventional SMA mix and for RAP replacement with and without elastomer modifier is found to be 28 and 25 blows each side respectively. The results for ITS and TSR for conventional SMA mix and SMA mix with optimized RAP with and without elastomer modifier are tabulated in Table 9.

Table 9 Indirect tensile strength test results

\begin{tabular}{cccc}
\hline \multirow{2}{*}{ SMA mixes } & \multicolumn{2}{c}{ Indirect Tensile Strength (kPa) } & $\begin{array}{c}\text { Tensile } \\
\text { Strength } \\
\text { Ratio (\%) }\end{array}$ \\
\hline Conventional mix & 357.19 & Conditioned & $\begin{array}{c}\text { Stioned } \\
\text { Ran }\end{array}$ \\
30\% RAP & 675.68 & 311.55 & 813.22 \\
60\% RAP & 703.22 & 658.76 & 90.75 \\
\hline
\end{tabular}

The results show that the SMA mix with optimized RAP with elastomer modifier is found to more moisture susceptibility than conventional SMA mix and optimized RAP without elastomer modifier.

\subsection{Rutting characteristics}

The rutting tests are conducted on the conventional SMA mix as well as for the SMA mix with optimized RAP with and without elastomer modifier. The test is carried out to find out 
the resistance to deformation due to repeated wheel load for wearing course. For this test, the maximum and minimum layer thickness is considered which is $40 \mathrm{~mm}$ and $50 \mathrm{~mm}$ thick respectively, with tyre pressure of $549 \mathrm{kPa}\left(5.6 \mathrm{~kg} / \mathrm{cm}^{2}\right)$ for each specimen at a frequency of 25 passes/min and it is carried out at room temperature until 10000 passes or $12 \mathrm{~mm}$ rut depth. The graphs of $40 \mathrm{~mm}$ and $50 \mathrm{~mm}$ thick specimens are shown in Figure 1 and Figure 2 respectively.

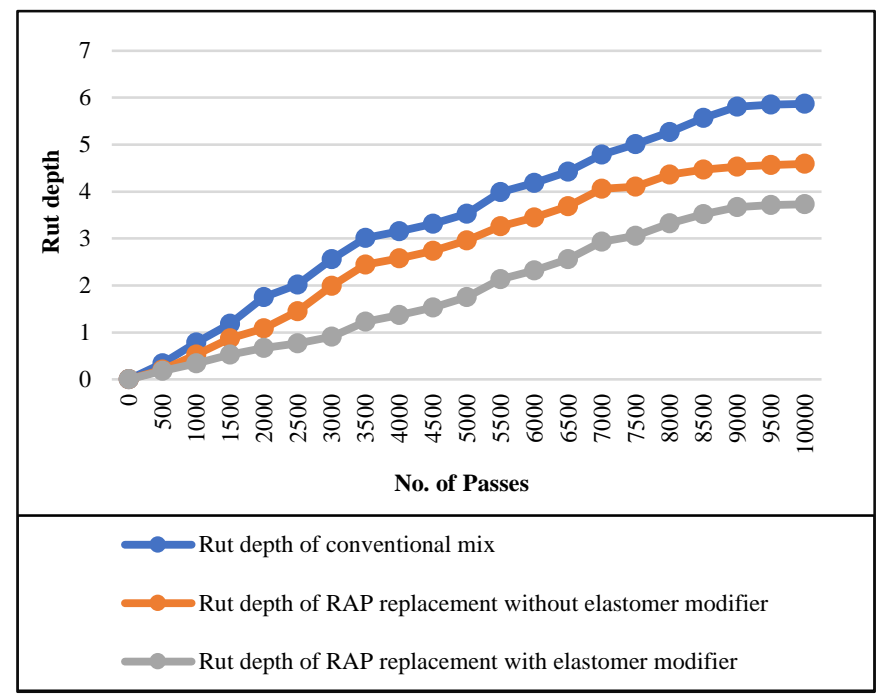

Figure 1. Rutting characteristics of 40mm thick SMA specimens

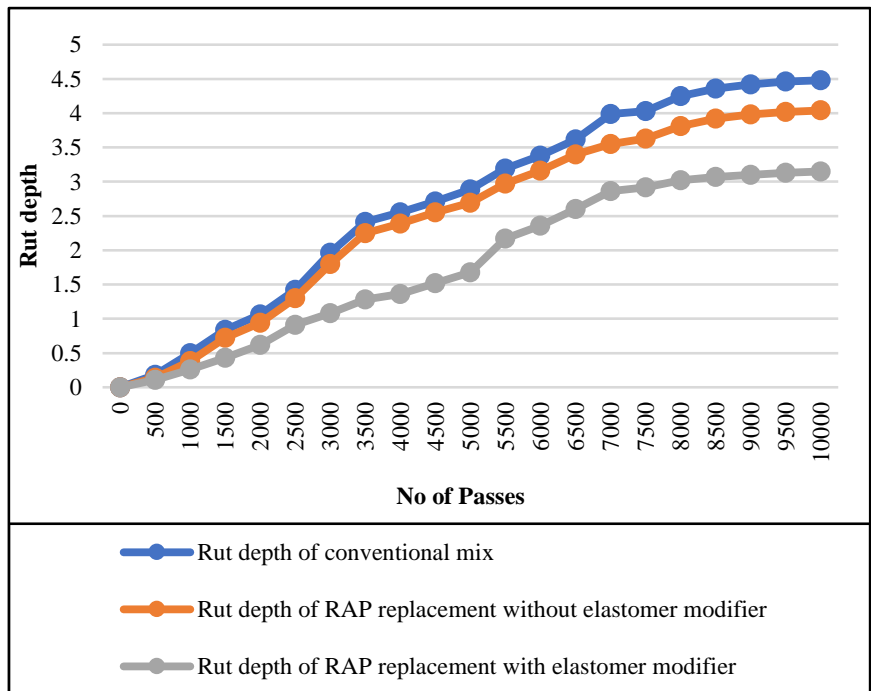

Figure 2. Rutting characteristics of 50mm thick SMA specimens

The rutting test is conducted for conventional mix and optimized RAP with and without elastomer modifier. The thickness of test specimens is $40 \mathrm{~mm}$ and $50 \mathrm{~mm}$. The result shows that for $40 \mathrm{~mm}$ thick beam the rut depth is 5.97, 4.59 and $3.73 \mathrm{~mm}$ respectively and for $50 \mathrm{~mm}$ thick beam $4.48,4.04$ and $3.15 \mathrm{~mm}$ respectively for different mix. Hence by adding elastomer modifier it increases the RAP content to $60 \%$ and decreases rut depth.

\subsection{Repeated load fatigue test}

The fatigue test is conducted on the conventional SMA mix and optimized RAP with and without the elastomer modifier. In this test marshall specimens of 32, 28 and 25 blows respectively and tested in four-point loading apparatus. The stress level and deformation are kept constant which is $10 \%$ and $5 \mathrm{~mm}$ respectively. The test was carried out to find out the resistance to deformation due to repeated wheel load. The test results are shown in Figure 3.

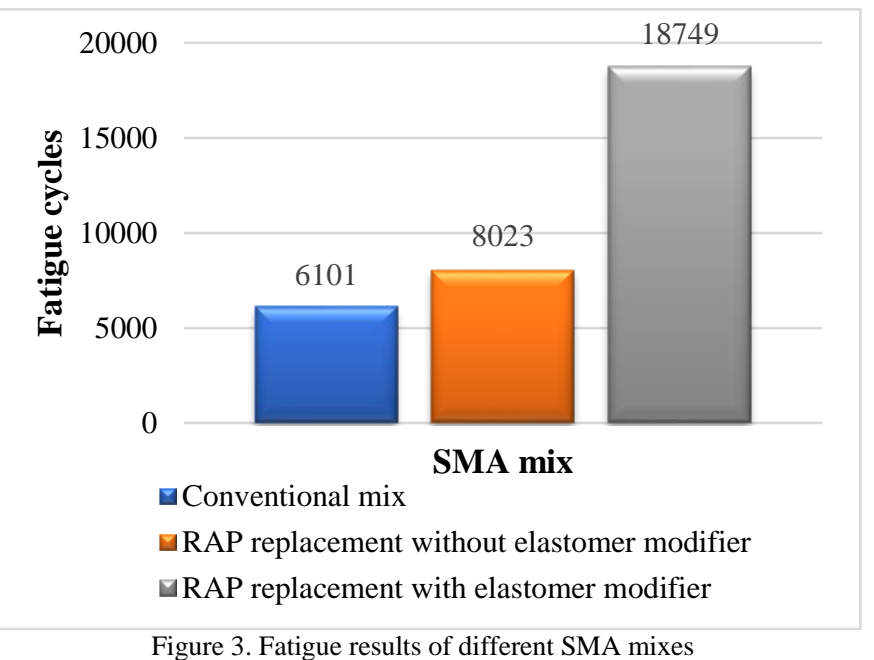

From the results obtained it is observed that the failure criteria for $5 \mathrm{~mm}$ deformation at $10 \%$ stress level shows that $60 \%$ RAP with the addition of elastomer modifier in SMA mix increases the fatigue life cycles compared to conventional mix and optimum RAP without elastomer modifier.

\section{CONCLUSIONS}

The physical tests on virgin aggregates, reclaimed asphalt pavement, baghouse dust and bitumen (VG-30) were carried out. Laboratory investigations on marshall mix specimens of varying percentages of RAP replacement with and without modifier was done. Performance tests like draindrown, moisture susceptibility, rutting and fatigue tests were conducted and the following conclusions were made:

1. The results obtained from basic laboratory tests of virgin aggregates, reclaimed asphalt pavement aggregates, baghouse dust and bitumen (VG-30) are within the specified limit as prescribed by MoRTH 500-35.

2. To determine the amount of RAP in the mix, marshall properties are determined. At 30\% RAP replacement, the stability value of the mix is found to be increased by 1.55 times than the conventional SMA mix. The obtained marshall properties are within the specified limits as per MoRTH.

3. By adding elastomer modifier, the optimum RAP is found to be $60 \%$ and marshall properties are within the specified values as prescribed in MoRTH.

4. The draindown test results yielded good results, the draindown of bitumen of the specimens was found out to be well within the limits i.e., maximum of $0.3 \%$ as prescribed in MoRTH.

5. The moisture susceptibility test results showed that SMA mix with60\% RAP with addition of elastomer modifier is found to be greater than the conventional SMA mix and SMA mix with $30 \%$ RAP without elastomer modifier. 
6. The rutting test is conducted for conventional SMA mix and RAP materials with and without elastomer modifier. The results show that by adding elastomer modifier it increases the RAP content up to $60 \%$ and decreases rut depth and hence it makes the mix rut resistant.

7. By conducting fatigue test for failure criteria of $5 \mathrm{~mm}$ deformation at $10 \%$ stress level shows that, SMA mix with $60 \%$ RAP with addition of elastomer modifier increases the fatigue life cycles compared to conventional mix and RAP replacement without elastomer modifier.

8. The performance test results have yielded better results for SMA mix with $60 \%$ RAP replacement with elastomer modifier compared to conventional SMA mix and SMA mix with $30 \%$ RAP. Hence, elastomer modifier can be stated as an effective alternate to conventional SMA mix since it results in appropriate use of waste materials and enhancement in performance properties.

\section{REFERENCES}

[1] Ashish Talati and Vaishakhi Talati (2014) "Study of Stone matrix asphalt for the flexible pavement", International Journal of Engineering Development and Research, Vol 2, Issue 1, ISSN: 23219939.

[2] Goutham Sarang, B.M. Lekha and J.S.Geethu (2015) "Laboratory performance of stone matrix asphalt mix with two aggregate gradation", J.Mod. Transport, Volume 23, Issue 2, Pg.130-136.

[3] Teja Tallam, Katasani Swetha and Dr. Ramesh A (2016) "Assessment of Stone mastic asphalt performance with the inclusion of fiber material on resilient characteristics", International Journal of Engineering Development and Research, IIT - Bombay.

[4] Marta Wasilewska (2017) "Evaluation of Skid resistance of wearing course of stone mastic asphalt mix in laboratory conditions", Materials science and Engineering, Volume 245.

[5] Anil Pradyumna T and Dr P K Jain (2014) "Use of RAP stabilized by hot mix recycling agents in bituminous road construction", Transportation Planning and Implementation Methodologies for Developing Countries (TPMDC), 10-12 December, Mumbai, India.

[6] Ahmed Mohamady, Ashraf Elshahat, Mahmoud Fathy AbdElmaksoud and Mohamed Hoseny Abdallah (2014) "Effect of using RAP on asphalt mix performance", IOSR Journal of Computer Engineering, ISSN: 2278-0661, Vol 16, Issue 6, Pg. 55-67.

[7] Dr. H S Jagadeesh and Mruthyunjay Kadayyanvarmath (2017) "Studies on SMA mix with partial RAP", International Journal of Science and Research (IJSR), ISSN: 2319-7064, Vol 6, Issue 8, Pg.6573.

[8] G.Valdes-Vidal, A.Calabi-Floody and E.Sanchez-Alonso (2018) "Performance evaluation of warm mix asphalt involving natural zeolite and reclaimed asphalt pavement for sustainable pavement construction", Construction and Building Materials, Vol 174, Pg. 576585 .

[9] Ratnasamy Muniandy and Bujang B.K. Huat (2006) "Laboratory diameteral Fatigue performance of stone matrix asphalt with cellulose oil palm fiber", American Journal of Applied Sciences, ISSN: 15469239, Vol 9, Issue 3, Pg. 2005-2010.

[10] Mohanlal Chandrawal, Anand Shankar Pandey and Dr.Tare.V (2016) "A Study of Rutting characteristics of Conventional and Modified bituminous concrete mix", IOSR Journal of Engineering, ISSN: 22503021, Vol 06, Issue 11, November, Pg. 34-47.

[11] Dr. Ganesh K and Sunil Kumar.V.Beli. (2013) "Studies on effect of wheel configuration temperature and type of binder on rutting characteristics of bituminous concrete mix", International Journal of Innovations in Engineering and Technology, ISSN: 2319-1058, Vol. 2, Issue 1.

[12] Adam Liphardt, Jan Krol and Piotr Radziszewski (2016) "Influence of polymer modified binder content from RAP on stone mastic asphalt rutting resistance", Theoretical Foundation of Civil Engineering, Pg. 407-413.

[13] Shivangi Gupta and A Veeraragavan, Fatigue behaviour of polymer modified bituminous concrete mixtures, Journal of the Indian Roads Congress, January-March 2009.
[14] Taher Baghaee Moghaddam, Mohamed Rehan Karim and Mahrez Abdelaziz (2011) "A review on fatigue and rutting performance of asphalt", Scientific Research and Essays, (Center for Transportation Research), 2011: 670-682.

[15] C Saba Toth and Zoltan (2015) "The effect of VIATOP $®$ plus FEP on the stiffness and low temperature behavior of hot mix asphalts", Journal of Silicate Based and Composite Materials, Pg. 126-131.

[16] Ministry of road transport \& highways, Specifications for road and bridge works (Fifth Revision), April 2013.

[17] IRC: SP: 79-2008, 'Tentative Specifications for Stone Matrix Asphalt', Indian Road Congress, New Delhi.

[18] IRC: SP: 73-2018, 'Manual of Specifications and standard for two laning highways with paved shoulder', Indian Road Congress, New Delhi.

[19] IRC: 120-2015, 'Recommended practice for recycling of bituminous pavements', Indian Road Congress, New Delhi.

[20] J. RETTENMAIER\& SOHNE,Technical Manual for Laboratory Tests. Rosenberg : Business Unit Road Construction, 2014.

[21] S. K. Khanna and C.E.G Justo, Highway materials and pavement testing laboratory Manual.

[22] S. K. Khanna, C.E.G Justo and A Veeraragavan, Highway Engineering, Revised 10th Edition. 